
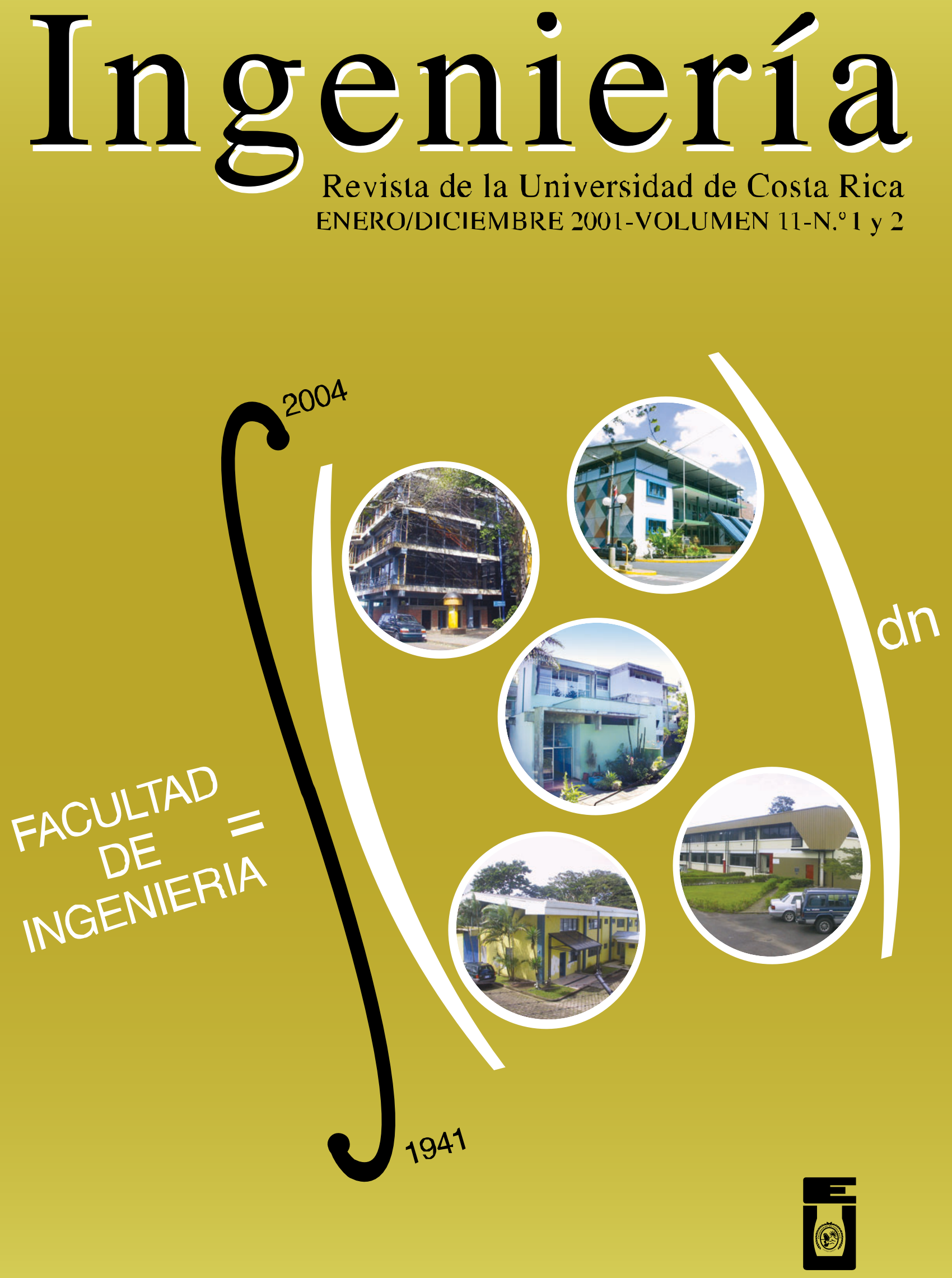


\title{
RECUPERANDO LA PÉRDIDA DE MOVILIDAD EN MANIPULADORES SERIALES POR MEDIO DE LA TEORÍA DE TORNILLOS
}

Jaime Gallardo Alvarado ${ }^{1}$

José María Rico Martínez²

\begin{abstract}
Resumen
Un manipulador serial se encuentra en una configuración singular cuando su órgano terminal es incapaz de moverse con una velocidad arbitraria dentro del espacio de trabajo. Esta situación es en general indeseable y debe evitarse o eliminarse. La primera opción es fácil de realizar aunque conlleva una reducción del espacio de trabajo. La mayoría de los manipuladores deben ser capaces de operar en configuraciones singulares. Si la singularidad es inevitable, es necesario implementar un procedimiento que permita eliminar la singularidad en que se encuentra el manipulador serial para tratar de recuperar su completa movilidad. En este trabajo se muestra cómo el producto de Lie, una operación fundamental del álgebra de Lie que es isomórfica con el álgebra de tornillos, permite identificar los tornillos asociados a los pares cinemáticos del manipulador, responsables de provocar la singularidad. A diferencia de otros procedimientos reportados en la literatura, el aquí empleado es aplicable a manipuladores que pierden más de un grado de libertad.
\end{abstract}

\section{INTRODUCCIÓN}

Un manipulador serial redundante posee más grados de libertad que los estrictamente necesarios para colocar su órgano terminal dentro del espacio de trabajo. Gracias a los citados grados de libertad excedentes que conforman la conocida redundancia del manipulador, éste posee una alta capacidad de ser manipulable y en consecuencia es capaz de ejecutar trayectorias más sofisticadas que las realizables con un manipulador no redundante.

El análisis de velocidad directo, dado un conjunto de velocidades generalizadas, consiste en determinar cuál es el efecto final sobre el órgano terminal. Por su parte, el análisis de velocidad inverso, suponiendo las condiciones de velocidad sobre el órgano terminal, consiste en determinar cuáles deben ser las velocidades generalizadas necesarias para satisfacer las condiciones de velocidad impuestas al órgano terminal.
Una singularidad ocurre cuando el órgano terminal de un manipulador serial es incapaz de moverse con una velocidad arbitraria dentro del espacio de trabajo. Esta situación conduce a una solución físicamente irrealizable del análisis de velocidad inverso.

Tradicionalmente, el análisis de singularidades, las llamadas "pesadillas cinemáticas" por Hollerbach, se ha estudiado extensamente, igualando a cero el determinante de la matriz Jacobiana. No obstante, debido a que la matriz Jacobiana de un manipulador redundante no es cuadrada, el cálculo de su determinante no tiene sentido.

A continuación, se resumen algunas contribuciones que abordan el tema de las singularidades en manipuladores seriales redundantes.

Baker y Wampler II (1988) desarrollaron un procedimiento basado en conceptos de topología matemática para analizar singularidades en 
manipuladores seriales redundantes que se componen exclusivamente de pares prismáticos o de "revoluta". El mismo Wampler II (1988) aplicó dichos conceptos en la determinación de las trayectorias factibles que permitan al manipulador abandonar la singularidad en que se encuentra. Ramdane-Cherif et al. (1996) resuelven el análisis de velocidad inverso mediante redes neuronales y de esta manera, analizan la redundancia del manipulador.

Sin duda, la teoría de tornillos infinitesimales es una alternativa confiable que permite de una forma sistemática y exacta explicar la naturaleza física de las singularidades.

Lipkin y Duffy (1985) mostraron que una singularidad está presente cuando la "cilindroide", generada entre dos tornillos coaxiales, degenera en una línea.

Cuando el determinante de una matriz es cero, condición necesaria para la existencia de la singularidad en manipuladores seriales no redundantes, existe dependencia lineal entre las columnas que la componen. Esta situación permite ubicar como una primera aproximación los posibles elementos que provocan la singularidad. Chevallier (1995) desarrolló un algoritmo basado en el álgebra de Lie para probar la dependencia lineal en conjuntos de tornillos. Podhorodeski et al. (1993) investigó la pérdida de movilidad en manipuladores redundantes agrupando los tornillos asociados a los pares cinemáticos del manipulador linealmente dependientes.

Es interesante mencionar que, si bien es cierto, existe una cantidad bastante respetable de contribuciones que tratan el problema de las singularidades en temas como la identificación y la caracterización de singularidades, sorprendentemente, el tema de escape de singularidades ha llamado poco la atención.

El primer estudio formal que trata del tema del escape de singularidades se debe a Hunt (1986), quien recurrió a la matriz de cofactores de la matriz Jacobiana y a la teoría de sistemas de tornillos para detectar la singularidad y al tornillo responsable de causarla. Cuando un manipulador serial no redundante pierde un grado de libertad, el rango de la correspondiente matriz Jacobiana es 5, por lo tanto se tienen cinco tornillos linealmente independientes con un tornillo recíproco común. Hunt (1986) determinó que en una configuración singular, el tornillo que es linealmente dependiente, no interviene en la determinación del tornillo que es recíproco. Un resultado similar fue reportado previamente por Sugimoto et al. (1982).

Parikian (1996) empleó los determinantes de la matriz Jacobiana y la matriz Gramiana para determinar la proximidad de singularidades en manipuladores seriales no redundantes. La combinación de los gradientes de esas matrices provee información para liberar al manipulador de la singularidad en que se encuentra. En ese mismo año, Karger (1996) utilizó el álgebra de Lie para describir las posibles configuraciones singulares en manipuladores seriales no redundantes.

En un trabajo previo Rico et al. (1995) introdujeron un método para identificar a los tornillos que provocan dicha singularidad, dado un manipulador serial no redundante en una configuración singular. A diferencia de los procedimientos anteriormente mencionados, en particular el de Hunt (1986). El método propuesto por Rico et al. (1995) es aplicable a manipuladores no redundantes que pierden más de un grado de libertad, tarea que se considera además de compleja, extremadamente laboriosa.

En este trabajo se extiende el método introducido por Rico et al. (1995), aplicable en manipuladores seriales no redundantes a los manipuladores seriales redundantes.

\section{CONCEPTOS PRELIMINARES}

Supongamos dos cuerpos rígidos que

$$
\$=\left[\begin{array}{l}
\mathrm{S} \\
\mathrm{so}
\end{array}\right]
$$


se encuentran conectados por medio de un par helicoidal \$. En coordenadas de Plucker, se representa en términos de una componente primaria $\mathbf{s}$ y una componente dual $\mathbf{s}_{\mathrm{O}}$ de la siguiente manera:

Donde $\mathbf{s}$ es un vector unitario a lo largo del eje instantáneo del par helicoidal, mientras que $\mathbf{s}_{\mathrm{O}}$ es el momento producido por un punto arbitrario $\mathrm{O}$, fijo a uno de los dos cuerpos, el cual se determina en términos del paso $\mathrm{h}$ del tornillo como

$$
\text { so }=h s+\text { so } \times r
$$

Donde $\mathbf{r}$ es un vector que se inicia en un punto del eje instantáneo del tornillo que conecta a los dos cuerpos y termina en el punto $\mathrm{O}$, mientras que $\times$ representa al producto cruz usual del álgebra vectorial tridimensional.

A principios del siglo pasado Ball (1 900) definió el estado de velocidad de un cuerpo rígido cuando se observa desde otro cuerpo o sistema de referencia, como el arreglo de una componente primaria $\omega$ y una componente dual $\mathrm{vO}$ de la siguiente manera:

$$
\mathrm{V}=\left\lfloor\begin{array}{l}
\omega \\
\mathrm{vo}
\end{array}\right\rfloor
$$

Donde $\omega$ y vO son los vectores velocidad angular y velocidad de un punto $O$ fijo al cuerpo rígido, como se observan desde el sistema de referencia seleccionado.

El mismo Ball (1 900) definió el estado de velocidad como un giro infinitesimal sobre un tornillo, así el estado de velocidad, ecuación (3), puede reescribirse como:

$$
\mathrm{V}=\omega \$
$$

Se sabe que en un manipulador serial el estado de velocidad del órgano terminal, cuerpo $\mathrm{m}$, con respecto al eslabón base, cuerpo 0 , puede escri- birse como una combinación lineal de los tornillos infinitesimales asociados a los pares cinemáticos que conforman al manipulador como

$$
\omega_{1} \$_{1}+\omega_{2} \$_{2}+\ldots+\omega_{m-1} \$_{m-1}+\omega_{m} \$_{m}={ }^{0} \mathrm{~V}^{\mathrm{m}}
$$

donde $\omega$ i representa el cambio instantáneo de velocidad relativo entre dos cuerpos adyacentes.

Por lo tanto, reescribiendo en forma matricial la ecuación (5), el análisis de velocidad directo se resuelve mediante la expresión:

$$
\left[\begin{array}{lllll}
\$_{1} & \$_{2} & \ldots & \$_{m-1} & \$_{m}
\end{array}\right]\left\lfloor\begin{array}{l}
\omega_{1} \\
\omega_{2} \\
\cdots \\
\omega_{m-1} \\
\omega_{m}
\end{array}\right\rfloor={ }^{0} \mathrm{~V}^{\mathrm{m}}
$$

Por su parte, el análisis de velocidad inverso se resuelve mediante:

$$
\left[\begin{array}{l}
\omega_{1} \\
\omega_{2} \\
\cdots \\
\omega_{m-1} \\
\omega_{m}
\end{array}\right]=\mathrm{J}^{-1} \quad{ }^{0} \mathrm{~V}^{\mathrm{m}}
$$

Donde $\mathrm{J}$ es la matriz Jacobiana que se forma por el subespacio generado por los tonillos $\$ 1, \$ 2$, $\ldots, \$ \mathrm{~m}-1$ y $\$ \mathrm{~m}$.

Cuando la matriz Jacobiana es singular, el manipulador serial se encuentra en una configuración singular; sin embargo, es evidente que para el análisis de velocidad inverso, si la matriz Jacobiana no es cuadrada, el cálculo de su inversa 
carece de sentido. Por lo tanto, es necesario recurrir a un procedimiento alterno que permita detectar las posibles singularidades.

Yoshikawa (1 985) definió al índice de manipulabilidad $\varpi$ como:

$$
\varpi=\sqrt{\mathrm{J} \mathrm{J}^{\mathrm{T}}}
$$

Donde T denota la transpuesta de la matriz. Es evidente que en una configuración singular el índice de manipulabilidad es nulo.

Una vez que la singularidad se ha detectado, es necesario identificar el tornillo o tornillos infinitesimales causantes de la singularidad a fin de que el manipulador recupere su movilidad. En el trabajo de Rico et al. (1 995) se muestra que el producto de Lie, una operación fundamental del álgebra de Lie, es una herramienta eficaz para identificar los tornillos responsables de provocar singularidades en manipuladores seriales no redundantes.

Sean $\$ 1=(\mathbf{s} 1, \mathbf{s O} 1)$ y $\$ 2=(\mathbf{s} 2, \mathbf{s O} 2)$ dos elementos del álgebra de Lie (3). El producto de Lie, [\$1 \$2] se define como:

$$
\left[\begin{array}{ll}
\$_{1} & \$_{2}
\end{array}\right]=\left[\begin{array}{l}
\mathrm{S}_{1} \times \mathrm{S}_{2} \\
\mathrm{~S}_{1} \times \mathrm{SO}_{2}-\mathrm{S}_{2} \times \mathrm{SO}_{1}
\end{array}\right]
$$

Por su parte, Hausner y Schwartz (1968) definen el producto de Lie en términos de desplazamientos Euclidianos:

$$
\left[\begin{array}{ll}
\$_{1} & \$_{2}
\end{array}\right]=\frac{\mathrm{d}}{\mathrm{dt}} \quad\left\{\mathrm{m}_{1}(\mathrm{t}) \$_{2}\left[\mathrm{~m}_{1}(\mathrm{t})^{-1}\right]\right\}_{t=0}
$$

Donde $\$ 1$ es un vector tangente al desplazamiento Euclidiano m1(t) en el tiempo $t=0$.

Puesto que para remover una singularidad en un manipulador serial es necesario que la trayectoria sea ortogonal y tangente a la dirección de la singularidad, por simple inspección de las expresiones (9) y (10) se deduce que el producto de Lie posee las características necesarias para tratar de efectuar un escape de la configuración singular en que se encuentra el manipulador.

\section{FUNDAMENTOS DEL MÉTODO}

Esta sección se inicia con una definición formal del concepto de singularidad en manipuladores seriales, este concepto puede analizarse desde diferentes perspectivas, todas ellas válidas e igualmente útiles.

Los siguientes conceptos son equivalentes:

- Un manipulador serial se encuentra en una configuración singular.

- El análisis de velocidad inverso requiere de una solución físicamente imposible de realizar.

- $\quad$ El índice de manipulabilidad $\varpi$ es cero.

- Si el manipulador serial es no redundante, el determinante de la matriz Jacobiana es cero o equivalentemente, las columnas de la matriz Jacobiana son linealmente dependientes.

Puesto que los manipuladores seriales no redundantes son casos particulares de los manipuladores seriales redundantes, es evidente que un procedimiento que permita eliminar singularidades en estos últimos también será válido para los primeros.

Consideremos un manipulador serial redundante, en el que el cuerpo designado como 0 es el eslabón base y el cuerpo designado como $m$ es el órgano terminal.

Sea $S=\{\$ 1, \$ 2, \ldots, \$ m-1, \$ m\}$, al conjunto $S$ se le denomina conjunto ordenado de tornillos. En particular, el tornillo $\$ 1$ es el que conecta el eslabón base con la cadena serial móvil y el tornillo \$m es el que conecta el órgano terminal con el resto de la cadena serial. 
Sea $\mathrm{SS}=\{\$ \mathrm{j}, \ldots, \$ \mathrm{k}\}$ un subconjunto de $\mathrm{S}$, con $\mathrm{k}>\mathrm{j} \mathrm{y} \mathrm{j} \geq 1, \mathrm{k} \leq \mathrm{m}$. Si los elementos de SS son adyacentes, entonces SS es un subconjunto ordenado de $\mathbf{S}$, que se denota como SOS.

Puesto que la dimensión máxima del conjunto ordenado de tornillos $\mathrm{S}$ es 6 , el conjunto $\mathrm{S}$ se divide en varios subconjuntos ordenados cuya cardinalidad máxima es de 6.

Se dice que el i-ésimo subconjunto ordenado de $\mathrm{S}, \mathrm{SOS}(\mathrm{i})$, es un subconjunto ordenado singular de S, SSOS(i), si:

$$
\operatorname{dim}[\operatorname{Sos}(\mathrm{i})] \leq \mathrm{k}-\mathrm{j}
$$

Adicionalmente, la diferencia

$(k-j+1)-\operatorname{dim}[S O S(i)]$ se conoce como los grados de libertad que ha perdido el subconjunto ordenado.

Un subconjunto ordenado singular es un subconjunto ordenado singular mínimo SOSM(i), si el subconjunto ordenado singular pierde como máximo un grado de libertad y si su cardinalidad es la menor posible para provocar este grado de libertad perdido. A fin de identificar los tornillos que son linealmente independientes dentro del subconjunto ordenado singular mínimo, es necesario determinar los subconjuntos de éste que son singulares, sin necesidad de ser ordenados. Un subconjunto singular de un subconjunto ordenado singular mínimo, es un subconjunto singular mínimo SSM(i) si éste pierde como máximo un grado de libertad y su cardinalidad es la menor posible para provocar la singularidad.

Es razonable suponer que en una configuración singular, el subconjunto ordenado singular mínimo contiene al tornillo causante de por lo menos una singularidad. Por lo tanto, analizando los diferentes subconjuntos de un subconjunto ordenado singular mínimo y su dimensión, es posible identificar al tornillo asociado a la singularidad. Es importante mencionar que en este punto no es necesario que los elementos de un subconjunto del subconjunto ordenado singular mínimo sean adyacentes.
En Rico et al. (1 995) se introducen las condiciones necesarias para eliminar la dependencia lineal en el conjunto ordenado de tornillos, éstas se resumen y en su caso se adecuan al presente trabajo.

Sea $\mathrm{S}$ el conjunto ordenado de tornillos de un manipulador serial que se encuentra en una configuración singular:

- En Waldron et al. (1985) se indica que los tornillos extremos \$1 y \$m no afectan las posiciones relativas entre los restantes tornillos, por lo tanto, cualquier desplazamiento alrededor de éstos no puede sacar al manipulador serial de la singularidad en que se encuentra ni pueden ser descartados de inmediato.

- $\quad$ Si se desea evitar configuraciones singulares es necesario que ningún subconjunto ordenado genere una "subálgebra" del álgebra de Lie. Un resultado similar fue reportado previamente por Hunt (1978).

- Un desplazamiento alrededor del tornillo $\$ \mathrm{j}$ afecta a cada uno de los tornillos $\$ \mathrm{k}$ con $\mathrm{k}>\mathrm{j}$

- $\quad \operatorname{Si} \$ \mathrm{j}(\mathrm{t})$ y $\$ \mathrm{k}(\mathrm{t})$ junto con $\mathrm{k}>\mathrm{j}$ son elementos del conjunto ordenado de tornillos en el tiempo $\mathrm{t}=0$, el tornillo $\$ \mathrm{k}(\Delta \mathrm{t})$ puede calcularse aproximadamente como:

$$
\begin{aligned}
& \$ \mathrm{k}(\Delta \mathrm{t}) \approx \$ \mathrm{k}(0)+[\$ \mathrm{j}(0) \$ \mathrm{k}(0)] \Delta \mathrm{t} \\
& =\$ \mathrm{k}+\left[\begin{array}{ll}
\$ \mathrm{j} & \$ \mathrm{k}
\end{array}\right] \Delta \mathrm{t}
\end{aligned}
$$

- Supongamos los elementos \$f(i) y $\$ \mathrm{~h}(\mathrm{i})$, junto con $\mathrm{h}>\mathrm{f}$, que pertenecen al i-ésimo subconjunto ordenado singular mínimo SOSM(i). Si \$h(i) es, además, un elemento del subconjunto singular mínimo asociado al subconjunto ordenado singular mínimo, entonces, existen elementos que resultan de los diferentes productos de Lie presentes entre las combinaciones de los elementos del subconjunto ordenado singular mínimo con su subconjunto singular mínimo, tales que algunos de ellos no pertenecen al subespacio generado por el subconjunto ordenado singular mínimo. 
- La dimensión del subespacio generado por el subconjunto ordenado singular mínimo que se obtiene al sustituir \$h(i)+[\$f(i) \$h(i)] en lugar de \$h(i), es equivalente a la cardinalidad del subconjunto ordenado singular mínimo. De esta manera, con la sustitución el subconjunto ordenado singular mínimo deja de ser singular. Al tornillo \$f(i) se le denomina actuador y al tornillo $\$$ h(i) se le denomina receptor. Los desplazamientos de los tornillos asociados a la singularidad no afectan a la dimensión de los subconjuntos de los restantes tornillos.

- Los productos de Lie que no se encuentran en el subespacio generado por el subconjunto ordenado singular mínimo, contienen los tornillos causantes de la singularidad. De esta manera, por inspección de esos productos de Lie, se identifican los tornillos actuador y receptor.

- Un tornillo actuador puede restaurar la dimensión de más de un subconjunto ordenado singular mínimo. Una vez que se ha restaurado la dimensión de cada uno de los subconjuntos ordenados singulares mínimos, resulta evidente que el manipulador serial se encuentra fuera de la configuración singular y con ello recupera su completa movilidad.

\subsection{UN ALGORITMO PARA ELIMINAR SINGULARIDADES}

En esta sección se proporcionan los pasos necesarios para restaurar la dimensión de cada uno de los subconjuntos ordenados singulares mínimos, en el caso de que un manipulador serial se encuentre en una configuración singular.

1. Determine en coordenadas de Plucker los tornillos infinitesimales asociados a los pares cinemáticos que conforman al manipulador se-rial.

2. Determine el conjunto ordenado de los tornillos S.
3. Calcule el determinante del producto de la matriz Jacobiana por su transpuesta, si éste es cero, entonces el manipulador serial se encuentra en una configuración singular.

4. Identifique y descarte los tornillos que no son los responsables de la singularidad.

5. Determine un subconjunto ordenado singular mínimo y su correspondiente subconjunto singular mínimo.

6. Calcule los productos de Lie resultantes de combinar cada uno de los elementos del subconjunto ordenado singular mínimo con los del subconjunto singular mínimo, tomando en cuenta que los subíndices de los posibles tornillos actuadores son inferiores a los de los receptores.

7. Seleccione los productos de Lie calculados en el punto 6 que no se encuentren en el subespacio generado por el subconjunto ordenado singular mínimo.

8. Identifique al tornillo asociado a la singularidad, inspeccionando los productos de Lie seleccionados en el punto 7 .

9. Determine el nuevo "subconjunto ordenado singular mínimo", calculando los correspondientes productos de Lie y determine su nueva dimensión.

Una vez que se han completado satisfactoriamente los 9 pasos indicados para cada uno de los subconjuntos ordenados singulares mínimos, el manipulador se encuentra fuera de la configuración singular y con ello ha recuperado su completa movilidad.

\section{EJEMPLOS}

Para mostrar la eficiencia de la metodología seleccionada en esta sección se analizan tres ejemplos de manipuladores seriales redundantes, los cuales se encuentran intencionalmente en configuraciones singulares. 


\subsection{MANIPULADOR SERIAL DE SIETE GRADOS DE LIBERTAD}

Considere el manipulador serial redundante que se muestra en la figura 1 .

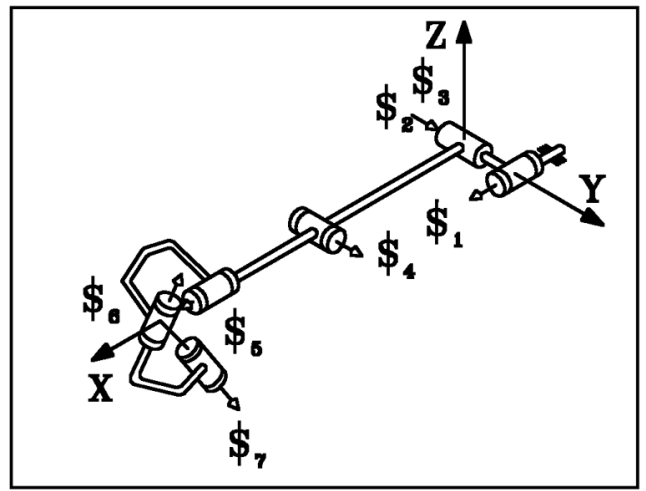

Figura 1: Manipulador RCRRRR

Los tornillos infinitesimales en coordenadas de Plucker, asociados a los pares cinemáticos que conforman al manipulador serial vienen dados por:

$\$ 1=(1,0,0 ; 0,0,-1)$,

$\$ 2=(0,0,0 ; 0,1,0)$,

$\$ 3=(0,1,0 ; 0,0,0)$,

$\$ 4=(0,1,0 ; 0,0,5)$,

$\$ 5=(1,0,0 ; 0,0,0)$,

$\$ 6=(0,0.7071,0.7071 ; 0,-7.071,7.071)$, $\$ 7=(0,0.7071,-0.7071,0,7.071,7.071)$,

donde los tornillos \$2 y \$3 están asociados al par cilíndrico.

El conjunto ordenado de tornillos $\mathrm{S}$ del manipulador viene dado por:

\section{$S=\{\$ 1, \$ 2, \$ 3, \$ 4, \$ 5, \$ 6, \$ 7\}$}

El determinante del producto de la matriz Jacobiana $\mathrm{J}=\left[\begin{array}{lllllll}\$ 1 & \$ 2 & \$ 3 & \$ 4 & \$ 5 & \$ 6 & \$ 7\end{array}\right]$ por su transpuesta es cero, por lo tanto el manipulador se encuentra en una configuración singular.

Los tornillos extremos del manipulador son $\$ 1$ y $\$ 7$. Los elementos del grupo $\{\$ 5, \$ 6, \$ 7\}$ son concurrentes y generan una subálgebra de e(3), ésta corresponde al subgrupo de rotaciones del grupo Euclideo E(3). Los tornillos \$1, \$2, \$5, \$6 y $\$ 7$ no son los causantes de provocar la singularidad y pueden descartarse de inmediato.

Por inspección, un subconjunto ordenado singular mínimo viene dado por:

$\operatorname{SOSM}(1)==\{\$ 1, \$ 2, \$ 3, \$ 4, \$ 5\}$,

y su correspondiente subconjunto singular mínimo viene dado por:

$\operatorname{SSM}(1)==\{\$ 1, \$ 3, \$ 4, \$ 5\}$.

Por lo tanto, es necesario considerar los siguientes productos de Lie:

$\left[\begin{array}{ll}\$ 2 & \$ 3\end{array}\right],\left[\begin{array}{ll}\$ 2 & \$ 4\end{array}\right],\left[\begin{array}{ll}\$ 2 & \$ 5\end{array}\right],\left[\begin{array}{ll}\$ 3 & \$ 4\end{array}\right],\left[\begin{array}{ll}\$ 3 & \$ 5\end{array}\right]$ y $[\$ 4 \$ 5]$.

Sin embargo, sólo los productos de Lie [ $\begin{array}{ll}\$ 3 & \$ \text { ], }\end{array}$ [ $\left.\begin{array}{ll}\$ 3 & \$ 5\end{array}\right]$ y [ $\$ 4$ \$5] no pertenecen al subespacio generado por el subconjunto ordenado singular mínimo SOSM(1), por consiguiente, los tornillos causantes de la singularidad pueden ser $\$ 3$ y $\$ 4$.

La sustitución de $\$ 5+[\$ 4 \$ 5] \Delta t$ en lugar de $\$ 5$ en el subconjunto ordenado singular mínimo $\operatorname{SOSM}(1)$ conduce a:

$\operatorname{dim}\left[\begin{array}{lllll}\$ 1 & \$ 2 & \$ 3 & \$ 4 & \$ 5+\left[\begin{array}{ll}\$ 4 & \$ 5\end{array}\right] \Delta t\end{array}\right]=5$,

mientras que la sustitución de $\$ 4+\left[\begin{array}{ll}\$ 3 & \$ 4\end{array}\right] \Delta t$ en lugar de $\$ 4$ y de $\$ 5+\left[\begin{array}{ll}\$ 3 & \$ 5\end{array}\right] \Delta \mathrm{t}$ en lugar de $\$ 5$, ambas en el subconjunto ordenado singular mínimo $\operatorname{SOSM}(1)$, conduce a:

$\operatorname{dim}\left[\begin{array}{llll}\$ 1 & \$ 2 & \$ 3 & \$ 4+\left[\begin{array}{ll}\$ 3 & \$ 4\end{array}\right] \Delta t \quad \$ 5+\left[\begin{array}{ll}\$ 3 & \$ 5\end{array}\right] \Delta t\end{array}\right]=5$.

Estos resultados indican que cualquiera de los tornillos actuadores $\$ 3$ o $\$ 4$, pueden restaurar la dimensión del subconjunto ordenado singular mínimo SOSM(1). Sin embargo,

$\operatorname{dim}\left[\begin{array}{llllll}\$ 2 & \$ 3 & \$ 4 & \$ 5+\left[\begin{array}{llll}\$ 4 & \$ 5\end{array}\right] \Delta \mathrm{t} & \$ 6 & \$ 7\end{array}\right]=5$, $\mathrm{y}$

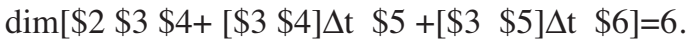


Por consiguiente, el manipulador puede abandonar la configuración singular en que se encuentra si el conjunto ordenado de tornillos original S se sustituye por:

\section{$\left\{\$ 1, \$ 2, \$ 3, \$ 4+\left[\begin{array}{ll}\$ 3 & \$ 4\end{array}\right] \Delta \mathrm{t}, \$ 5+\left[\begin{array}{ll}\$ 3 & \$ 5\end{array}\right] \mathrm{t}, \$ 6, \$\right\}$}

de esta manera, a fin de que el manipulador recupere su completa movilidad es necesario actuar la revoluta $3, \$ 3$.

\subsection{EJEMPLO 2, MANIPULADOR DE 9 GRADOS DE LIBERTAD}

Considere el manipulador serial redundante que se muestra en la figura 2.

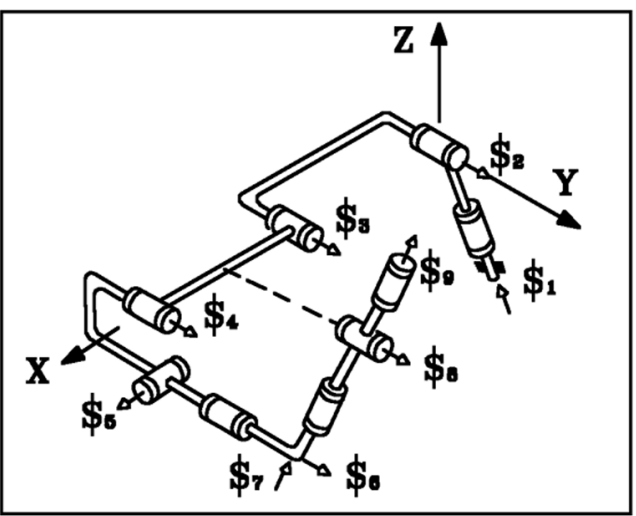

Figura 2: Manipulador RRRRRRRR

En coordenadas de Plucker, los tornillos infinitesimales asociados a los pares cinemáticos que conforman al manipulador vienen dados por:

$\$ 1=(0,7071 ; 0 ; 0,7071 ; 0 ; 0 ; 0)$,

$\$ 2=(0,1,0 ; 0,0,0)$,

$\$ 3=(0,1,0 ; 0,0,2)$,

$\$ 4=(0,1,0 ; 0,0,4)$,

$\$ 5=(1,0,0 ; 0,-1,0)$,

$\$ 6=(0,1,0 ; 1,0,4)$,

$\$ 7=(-0.7071,0,-0.7071 ; 1.4142,-2.121,1.4142)$,

$\$ 8=(0,1,0 ; 0,0,3)$,

$\$ 9=(-0,7071 ; 0 ; 0,7071 ; 1,4142 ;-2,121 ; 1,4142)$.
De esta manera, el conjunto ordenado de tornillos viene dado por:

$S=\{\$ 1, \$ 2, \$ 3, \$ 4, \$ 5, \$ 6, \$ 7, \$ 8, \$ 9\}$

El determinante del producto de la matriz Jacobiana, J=[ [ $\$ 1 \$ 2 \$ 3 \$ 4 \$ 5 \$ 6 \$ 7 \$ 89]$, por su transpuesta es cero, por lo tanto, el manipulador se encuentra en una configuración singular.

Los tornillos extremos del manipulador son $\$ 1$ y $\$ 9, \mathrm{y}$ puesto que no son los causantes de provocar la singularidad pueden ser descartados de inmediato.

Por inspección, un subconjunto ordenado singular mínimo viene dado por:

$\operatorname{SOSM}(1)==\{\$ 2, \$ 3, \$ 4\}$,

y su correspondiente subconjunto singular mínimo es el mismo subconjunto SOSM(1).

Por consiguiente, es necesario considerar los siguientes productos de Lie:

$\left[\begin{array}{ll}\$ 2 & \$ 3\end{array}\right],\left[\begin{array}{ll}\$ 2 & \$ 4\end{array}\right]$ y [ $\left.\$ 334\right]$,

que no pertenecen al subespacio generado por el subconjunto ordenado singular mínimo.

La sustitución de \$4+[ $\$ 3$ \$ $\$ 4 \Delta \mathrm{t} 1$ en lugar de $\$ 4$ en el subconjunto ordenado singular mínimo $\operatorname{SOSM}(1)$, conduce a:

$\operatorname{dim}\left[\begin{array}{llll}\$ 2 & \$ 3 & \$ 4 & \$ 5+\left[\begin{array}{ll}\$ 3 & \$ 4\end{array}\right] \Delta t 1\end{array}\right]=3$, y con ello el subconjunto $\operatorname{SOSM}(1)$ deja de ser singular.

Otro subconjunto ordenado singular mínimo viene dado por:

$\operatorname{SOSM}(2)=\{\$ 2, \$ 3, \$ 4+[\$ 3 \$ 4] \Delta t 1, \$ 5, \$ 6\}$,

y su correspondiente subconjunto singular mínimo viene dado por:

$\operatorname{SSM}(2)==\left\{\$ 2, \$ 3, \$ 4+\left[\begin{array}{ll}\$ 3 & \$ \Delta t 1, \$ 6\} .\end{array}\right.\right.$ 
Los resultados obtenidos para el primer subconjunto ordenado singular mínimo SOSM(1) indican que sólo es necesario considerar al producto de Lie [\$5 \$6] para el segundo subconjunto ordenado singular mínimo $\operatorname{SOSM}(2)$, el cual no se encuentra en el subespacio generado por el subconjunto $\operatorname{SOSM}(2)$.

Tomando en cuenta que el producto de Lie es nilpotente y sustituyendo $\$ 6+\left[\begin{array}{ll}\$ 5 & \$ 6\end{array}\right] \Delta \mathrm{t} 2$ en lugar de $\$ 6$ en el subconjunto $\operatorname{SOSM(2)}$ se tiene que:

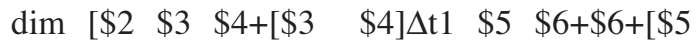
$\$ 6] \Delta \mathrm{t} 2]=5$.

De esta manera, con dicha sustitución el subconjuto $\operatorname{SOSM}(2)$ deja de ser singular.

Un tercer subconjunto ordenado singular mínimo $\operatorname{SOSM}(3)$ viene dado por:

$\operatorname{SOSM}(3)==\{\$ 7, \$ 8, \$ 9\}$,

y su correspondiente subconjunto singular mínimo $\operatorname{SSM}(3)$ viene dado por:

$\operatorname{SSM}(3)==\{\$ 7, \$ 9\}$.

Así, sólo es necesario considerar el producto de Lie [ \$8 \$9], el cual no se encuentra en el subespacio generado por $\operatorname{SOSM}(3)$.

La sustitución de \$9+[\$8 \$9t3 en lugar de \$9 conduce a:

$\operatorname{dim}\left[\begin{array}{lll}\$ 7 & \$ 8 & \$ 9+\left[\begin{array}{ll}\$ 8 & \$ 9\end{array}\right] \Delta \mathrm{t} 3\end{array}\right]=3$,

y con ello el subconjunto $\operatorname{SOSM(3)~deja~de~ser~}$ singular.

La colección de estos resultados indica que a fin de recuperar la completa movilidad del manipulador, el conjunto ordenado de tornillos original $\mathrm{S}$ debe sustituirse por:

$\mathrm{S}=\left\{\$ 1, \$ 2, \$ 3, \$ 4, \$ 4+\left[\begin{array}{l}\$ 3 \\ \$ 4\end{array}\right] \Delta \mathrm{t} 1\right.$,

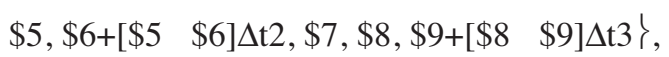

por lo que es necesario actuar las revolutas $3,5 \mathrm{y}$ 8.

\subsection{EJEMPLO 3, MANIPULADOR DE SIETE GRADOS DE LIBERTAD}

El manipulador serial redundante que se muestra en la figura 3 es una adaptación de una cadena cinemática cerrada propuesta por Sugimoto et al. (1982). El mecanismo original fue propuesto para su caracterización y los resultados obtenidos demuestran que se trata de una estructura.

Con el objeto de ejemplificar la metodología que se aplica en el presente trabajo, el mecanismo original se "rompió" para producir una cadena serial abierta y de esta manera, analizar las configuraciones singulares.

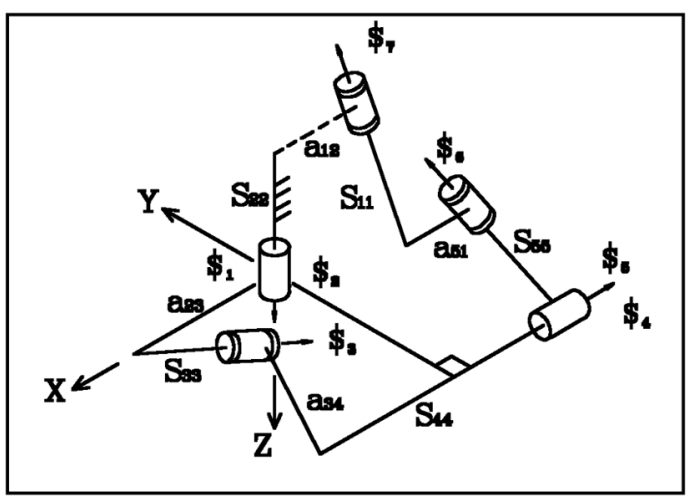

Figura 3: Manipulador RCRCR

Los parámetros del mecanismo vienen dados por:

a12 $=0.577$ u.1., a23=1 u.1., a34=2.828 u.1.,

a45=0 u.1., a51=1.154 u.1., S11=-2.804 u.1.,

$\mathrm{S} 33=2.828$ u.1., $\mathrm{S} 55=3$ u.1.,

$\alpha 12=150, \alpha 23=135, \quad \alpha 34=270, \quad \alpha 45=90$, $\alpha 51=330$,

donde los ángulos $\alpha$ están medidos en grados y u.l. significa unidades de longitud. 
En coordenadas de Plucker, los tornillos infinitesimales asociados a los pares cinemáticos que conforman al manipulador vienen dados por:

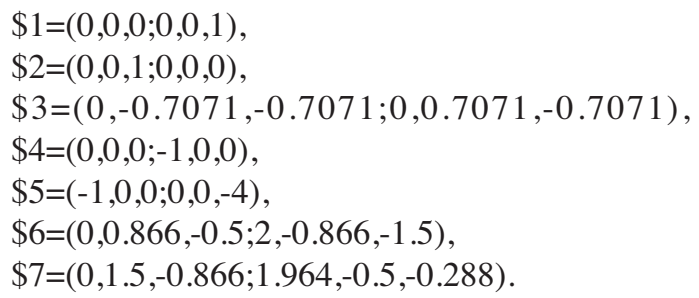

De esta manera, el conjunto ordenado de tornillos viene dado por:

$S=\{\$ 1, \$ 2, \$ 3, \$ 4, \$ 5, \$ 6, \$ 7\}$

El determinante del producto de la matriz Jacobiana, J=[ $\left.\begin{array}{lllllll}\$ 1 & \$ 2 & \$ 3 & \$ 4 & \$ 5 & \$ 6 & \$ 7\end{array}\right]$, por su transpuesta es cero, por consiguiente, el manipulador se encuentra en una configuración singular.

Los tornillos extremos del manipulador son $\$ 1$ y $\$ 7$, y puesto que no son los causantes de provocar la singularidad pueden ser descartados de inmediato.

Por inspección, existe un único subconjunto ordenado singular mínimo, $\operatorname{SOSM}(1)$, el cual viene dado por:

$\operatorname{SOSM}(1)=\{\$ 1, \$ 2, \$ 3, \$ 4, \$ 5, \$ 6, \$ 7\}$

y su correspondiente subconjunto singular mínimo es el mismo subconjunto SOSM(1).

Por lo tanto, es necesario considerar los siguientes productos de Lie,

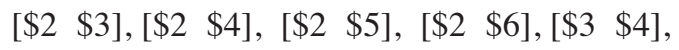

[\$3 \$5], [\$3 \$6], [\$4 \$5], [ $\$ 4 \$ 6$ y [ $\$$ \$5 \$6].

Los siguientes no pertenecen al subespacio generado por el subconjunto ordenado singular mínimo: $\left[\begin{array}{ll}\$ 2 & \$ 4\end{array}\right],\left[\begin{array}{ll}\$ 2 & \$ 5\end{array}\right],\left[\begin{array}{ll}\$ 2 & \$ 6\end{array}\right],\left[\begin{array}{ll}\$ 3 & \$ 4\end{array}\right],\left[\begin{array}{ll}\$ 3 & \$ 5\end{array}\right]$,

$\left[\begin{array}{ll}\$ 3 & \$ 6\end{array}\right],\left[\begin{array}{ll}\$ 4 & \$ 6\end{array}\right]$ y $\left[\begin{array}{ll}\$ & \$ 6\end{array}\right]$.

De esta manera, suponiendo que el tornillo actuador es $\$ 3$, tenemos que

$\operatorname{dim}$

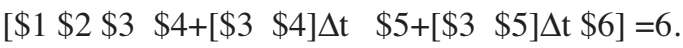

Además:

$\operatorname{dim}$

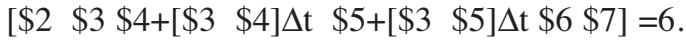

De este modo, actuando la tercer revoluta $\$ 3$, el manipulador puede abandonar la configuración singular en que se encuentra y con ello recupera su completa movilidad.

\section{CONCLUSIONES}

Si un manipulador serial redundante o no redundante, se encuentra en una configuración singular, en este trabajo se muestra cómo el producto de Lie permite identificar los tornillos causantes de la singularidad. La metodología seleccionada permite eliminar singularidades en manipuladores que pierden más de un grado de libertad, tarea que se considera compleja y extremadamente laboriosa. Finalmente, el método seleccionado se probó exitosamente en tres ejemplos.

\section{AGRADECIMIENTO}

El presente trabajo surgió como una sugerencia del Dr. José Alfonso Pámanes García, ProfesorInvestigador del Instituto Tecnológico de la Laguna, México.

\section{BIBLIOGRAFÍA}

1. Baker, D.R., y Wampler II, C.W., “'On the Inverse of Redundant Manipulators". The International Journal of Robotics Research, Vol. 7, No. 2, pp. 3-21, 1988. 
2. Ball, R.S., A Treatise on the Theory of Screws. Cambridge University Press: Cambridge, 1 900.

3. Chevallier, D.P., "Coordinate Free Criteria for Testing the Linear Dependence of the Sets of Screws". En: Computational Kinematics '95, Merlet, J.-P., and Ravani, B., eds., Kluwer Academic Publishers, pp. 1 - 10, 1995.

4. Hausner, M., y Schwartz, J.T., Lie groups, Lie algebras. Gordon and Breach, New York, pp. 50, 1968 .

5. Hunt, K.H., Kinematic Geometry of Mechanisms. Oxford University Press: Oxford, 1978.

6. Hunt, K.H., ' Special Configurations of Robot-Arms Via Screw Theory". Robotica, Vol. 4, pp. 171-179, 1986.

7. Karger, A., ' Singularity Analysis of SerialRobot Manipulators". $\underline{\text { ASME Journal of }}$ Mechanical Design, Vol. 118, No. 4, pp. 520525, 1996.

8. Lipkin, H., y Duffy, J., “The Elliptic Polarity of Screws". ASME Journal of Mechanisms, Transmissions, and Automation in Design, Vol. 107, pp. 377-387, 1985.

9. Parikian, T.F., “'Gradient-Based Kinematic Control of Non-Redundant Serial Robots in Special Configurations". En: Recent Advances in Robot Kinematics, Lenarcic, J., y ParentiCastelli, V., editores, Dordretch Kluwer Academic Publishers, pp. 77-86, 1996.

10. Podhorodeski, R.P., Goldenberg, A.A., y Fenton, R.G., " A Null-Space Solution of the Inverse Kinematics of Redundant Manipulators Based on a Decomposition of
Screws". ASME Journal of Mechanical Design, Vol. 115, pp. 530-539, 1993.

11. Ramdane-Cherif, A., Perdereau, V., y Drouin, M., "Penalty Approach for a Constrained Optimization to Solve on-line the Inverse Kinematic Problem of Redundant Manipulators". Proc. 1996 IEEE International Conference on Robotics and Automation, Vol. 1, pp. 133-138. Minneapolis, Minnesota, 1 996.

12. Rico, J.M., Gallardo, J., y Duffy, J., “A Determination of Singular Configurations, and Their Escapement from Singularities Using Lie Products". En: Computational Kinematics '95, Merlet, J.-P., y Ravani, B., editores. Dordrecht Kluwer Academic Publishers, pp. 143-152, 1995.

13. Sugimoto, K., Duffy, J., y Hunt, K.H., "Special Configurations of Spatial Mechanisms and Robot Arms". $\underline{\text { Mechanism }}$ and Machine Theory, Vol. 17, No. 2, pp. 119132, 1982.

14. Waldron, K.J, Wang, S.L., y Bolin, S.J., “A Study of the Jacobian Matrix of Serial Manipulators". ASME Journal of Mechaninsms, Transmissions, and Automation in Design, Vol. 107, No. 2, pp. 230-238, 1985.

15. Wampler II, C.W., “Winding Number Analysis of Invertible Workspaces for Redundant Manipulators". The International Journal of Robotics Research, Vol. 7, No. 5, pp. 22-31, 1983.

16. Yoshikawa, T., 'Dynamic Manipulabi-lity of Robot Manipulators". Journal of Robotic Systems, Vol. 2, No. 1, 113-124, 1985. 
